\section{GARFIELD-VTE-Register: Blick auf die isolierte distale Venenthrombose}

Schellong SM et al. Isolated Distal Deep Vein Thrombosis: Perspectives from the GARFIELD-VTE Registry. Thromb Haemost 2019; 19: 1675-1685

Die isolierte distale Venenthrombose (IDDVT) macht bis zur Hälfte aller DVTs der unteren Extremitäten aus. In dieser Untersuchung wurden die Art der Behandlung und Behandlungsergebnisse bei Patienten mit IDDVT im Vergleich zu anderen VTE-Patientenpopulationen, nämlich Patienten mit proximaler DVT und Lungenembolie, die alle im GARFIELD-VTE-Register eingeschlossen sind, verglichen.

Das GARFIELD-VTE (Global Anticoagulant Registry in the Field - Venous Thromboembolism) -Register ist eine internationale, multizentrische Beobachtungsstudie von Patienten mit neu diagnostizierter VTE, an der sich 415 Zentren in 28 Ländern beteiligen. Ergebnisse von Interesse sind die Inzidenz wiederkehrender VTE-Episoden, Gesamtmortalität, Blutungen (alle oder größere gemäß ISTH-Kriterien), Krebs (diagnostiziert mindestens 30 Tage nach Index-VTE), Myokardinfarkt/akutes Koronarsyndrom und Schlaganfall/TIA. Nicht erfasst wurden bisher die Ereignisraten nach Behandlungsstatus.

In der Subanalyse wurde untersucht, ob die Lokalisation der DVT mit bekannten VTE-provozierenden Faktoren interagiert. Patienten mit IDDVT und proximaler DVT (PVDT) und Lungenembolie (PE) wurden stratifiziert nach dem Vorliegen neuer, transienter provozierender, ISTH-definierter Faktoren: mindestens eine Operation, Krankenhauseinweisung, Schwangerschaft, Hormonersatztherapie/orale Kontrazeption, akute medizinische Erkrankung oder Trauma der unteren Extremität innerhalb von 3 Monaten, persistierender provozierender Faktor wie aktiver Krebs zu Studienbeginn oder keiner dieser provozierenden Faktoren. Darüber hinaus wurde die 1-Jahres-Inzidenz von Gesamt- mortalität, VTE-Rezidiv und schweren Blutungen bestimmt.

Von den etwas mehr als 10000 infrage kommenden Patientinnen und Patienten wurden 2145 als IDDVT-Patienten eingestuft (21,3\%), 3846 als PDVT- und 4097 als PE-Patienten (38,1\% und 40,6\%). Das mittlere Alter lag bei 58,6 $\pm 16,9$ Jahren und der mittlere BMI betrug $28,4 \pm 6,6 \mathrm{~kg} / \mathrm{m}^{2}$.

Bei den IDDVT-Patienten konnte häufiger eine Operation (14,6\%) oder ein Beintrauma (13,2\%) nachgewiesen werden als bei den PDVT-Patienten (11,0\% bzw. 8,7\%) und den PE-Patienten (12,7\% bzw. 4,5\%). Im Vergleich zu IDDVT-Patienten hatten Patienten mit PDVT oder PE hingegen häufiger aktiven Krebs (7,2\% vs. 9,9\% und $10,3 \%)$.

Die Ergebnisse zum Einfluss provozierender Faktoren auf das Risiko eines erneuten Auftretens bei IDDVT waren hingegen kontrovers. Fast alle Patienten (IDDVT, PDVT und PE) erhielten eine Antikoagulationstherapie. In den IDDVT-, PDVT- und PE- 
Gruppen betrug der Anteil der Patienten, die eine Antikoagulationstherapie erhielten, nach 6 Monaten $61,4 \%, 73,9 \%$ und $81,1 \%$. Nach 12 Monaten waren dies für die IDDVT-Gruppe $45,8 \%$ und für die PDVT- und PE-Gruppen 54,7\% und 61,9\%. Über 12 Monate war die Inzidenz von Gesamtmortalität und Krebs bei den IDDVT-Patienten signifikant niedriger als bei PDVT-Patienten $(p<0,0001)$. Die Inzidenzrate eines VTE-Rezidivs war ebenfalls signifikant geringer $(p=0,0298)$. Ebenso war das Risiko für Todesfälle und Krebserkrankungen bei Patienten mit IDDVT im
Vergleich zu PE signifikant niedriger (beide $p<0,05)$. Bei den IDDVT-Patienten kam es seltener zu Blutungen, allerdings war die Inzidenz schwerer Blutungen zwischen den Gruppen nicht signifikant.

\section{FAZIT}

Die Studie zeigt, dass die meisten IDDVT-Patienten sowie diejenigen mit PDVT und PE eine Antikoagulationstherapie erhalten, in vielen Fällen für mindestens 12 Monate. Die Studie zeigt auch, dass Todesfälle und Krebserkrankungen bei Patienten mit IDDVT im Vergleich zu den anderen Gruppen seltener auftraten und dass IDDVT häufiger mit neuen, transienten provozierenden Faktoren assoziiert ist, während PDVT und PE eher das Ergebnis persistierender provozierender Faktoren sind, so das Autorenteam.

Richard Kessing, Zeiskam 